\title{
HISTORIA DE LA RAQUIANESTESIA Y DE LA ANESTESIA EPIDURAL EN ESPAÑA
}

Victoria Gonzalo Rodríguez, MaDolores Rivero Martínez, Mariano Pérez Albacetel, Ana l. López López' y Alejandro Maluff Torres?.

Hospital Clínico Universitario de Valladolid. Valladolid. España.

'Hospital Universitario Virgen de la Arrixaca. Murcia. España.

Resumen.- OBJETIVO: Conocer como se desarrollaron en nuestro país las técnicas de la raquianestesia y la anestesia epidural, y cuales fueron las aportaciones de los urólogos españoles.

MÉTODOS: Hemos revisado los libros de Historia de la Medicina, de la Urología y de Anestesia, las publicaciones periódicas y las Tesis Doctorales que sobre este tema se realizaron en la época.

RESULTADOS: En buena parte del siglo XX aquellos que se dedicaban a la cirugía administraban también la anestesia. Es a partir del año 1900 cuando comienzan a desarrollarse la raquianestesia y la anestesia epidural siendo numerosas y fundamentales las aportaciones de los urólogos españoles como F. Rusca Doménech, J.M. Batrina, M. Barragán Bonet, R. Lozano Monzón, L. Guedea Calvo, Gil Vernet, Fidel Pagés Miravé, V. Sagarra Lascuraín, Gómez Ulla, etc. realizando publicaciones en revistas de prestigio, comunicaciones en congresos y tesis doctorales sobre la anestesia raquídea y epidural.

Palabras clave: Raquianestesia. Anestesia epidural. Historia de la Urología.

Summary.- OBJECTIVES: To show the beginning of spinal and epidural anesthesia in our country and the contributions of Spanish urologists.

METHODS: We reviewed books and writings of History of Medicine, Urology and Anesthesia and Doctoral thesis about spinal and epidural anesthesia.

RESULTS: In the 20th century, surgeons also gave the anesthetic drugs to the patients. Spinal and epidural anesthesia were used for the first time in 1900. A lot of Spanish urologists like F. Rusca Doménech, J.M. Batrina, M. Barragán Bonet, R. Lozano Monzón, L. Guedea Calvo, Gil Vernet, Fidel Pagés Miravé, V. Sagarra Lascuraín, Gómez Ulla, etc, did research, writings in scientific journals and Doctoral thesis about anesthesia.

Keywords: Spinal anesthesia. Epidural anesthesia. History of Urology. 


\section{INTRODUCCIÓN}

En buena parte del siglo $X X$, aquellos que se dedicaban a la cirugía, se encargaban también de administrar la anestesia antes de iniciar la correspondiente práctica operatoria. Son los años 1899 y 1901 cuando se realizan las primeras publicaciones sobre el empleo de la raquianestesia y la anestesia epidural respectivamente (Figura 1).

Estas técnicas anestésicas están hoy en día, en cirugía, consolidadas gracias a los perfeccionamientos introducidos en su método de aplicación, vía de acceso, dosis y calidad de los anestésicos a emplear.

En Urología, se utiliza en más de la mitad de las intervenciones quirúrgicas (cirugía prostática, vesical, genitales externos, etc.).

Sin embargo su aplicación no sólo se limita al ámbito quirúrgico, ya que la anestesia epidural es la técnica fundamental en las clínicas de dolor para poder mantener una analgesia continua.

\section{OBJETIVO}

Nuestro objetivo es conocer como se desarrolló la implantación de estas técnicas en nuestro país junto con las aportaciones que realizaron los urólogos españoles.

\section{MATERIAL Y MÉTODOS}

Revisamos las publicaciones periódicas, así como los libros de historia de la Medicina, de la Urología y de la Anestesia.

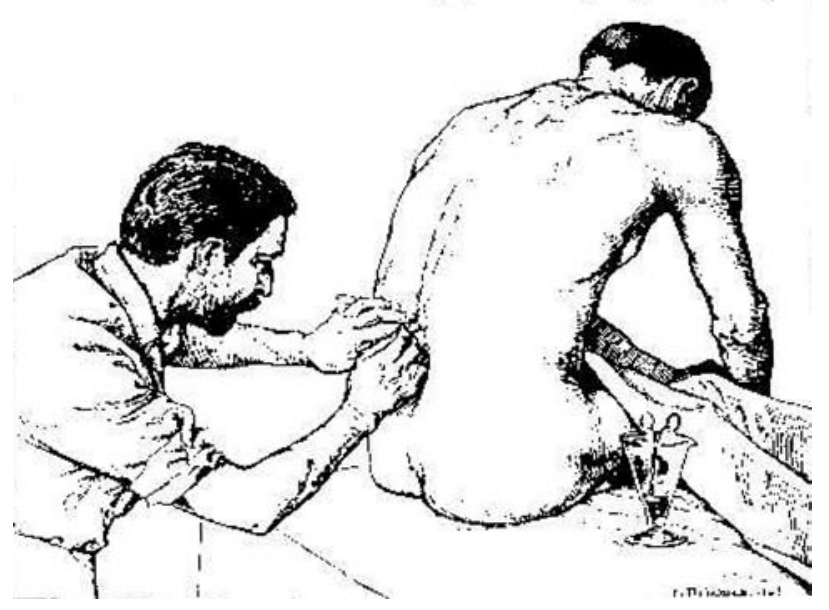

FIGURA 1. Colocación de paciente para la punción raquídea o epidural.

\section{RESULTADOS}

\section{RAQUIANESTESIA}

Las primeras noticias sobre la aplicación de la anestesia raquídea son las publicaciones en 1899 del cirujano alemán August Bier (1861-1949) y unos meses más tarde por el cirujano francés Theódore Tuffier (1861-1929). La publicación de estos trabajos en revistas de gran difusión y su presentación en el XIII Congreso internacional de Medicina en Paris en agosto de 1900 hacen que pronto cirujanos de todo el mundo ensayen estas técnicas (Figura 2).

En España las primeras publicaciones corresponden al cirujano catalán Francisco Rusca Doménech (1868-1909) en mayo de 1900, sobre 16 pacientes sometidos a raquianestesia presentando resultados satisfactorios (Figura 3 ).

De esta forma a lo largo de 1900 y 1901 son muchos los cirujanos que ensayan estas técnicas en sus clínicas, Manuel Barragán Bonet, Ricardo Lozano Monzón (Figura 4), Luis Guedea Calvo, Cordero Sora, etc.

A pesar de la rápida difusión de la anestesia raquídea, el empleo de la técnica disminuye entre lo años 1901 y 1904 debido a los efectos secundarios de la cocaína, hasta el momento el único anestésico empleado, denominándose la técnica "raquicocainización". Sin embargo la introducción de otras sustancias como la estovaína y la novocaína, menos tóxicas que la cocaína pero con un poder anestésico semejante, hacen de nuevo resurgir la raquianestesia.

A partir de 1914 debemos destacar varias escuelas en España entusiastas con el método de la
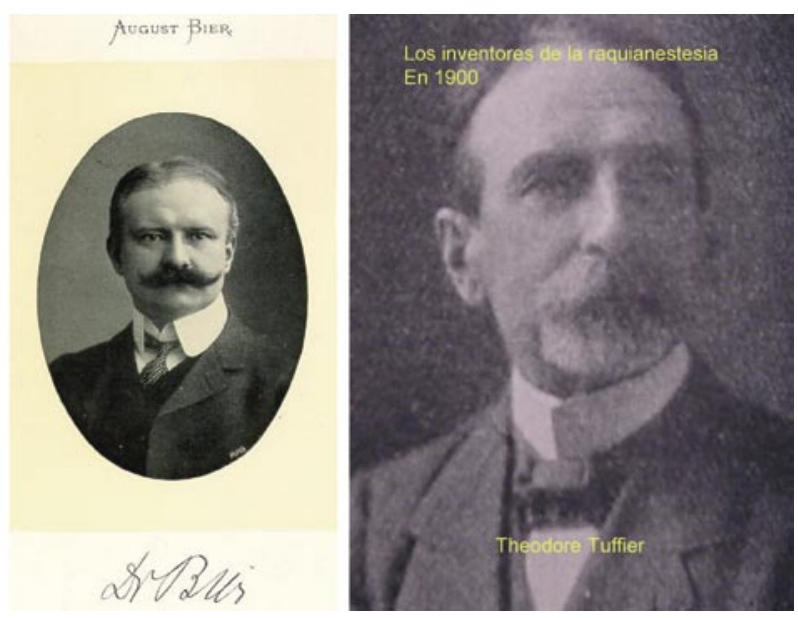

FIGURA 2. August Bier (1861-1949) y Theódore Tuffier (1867-1929). 


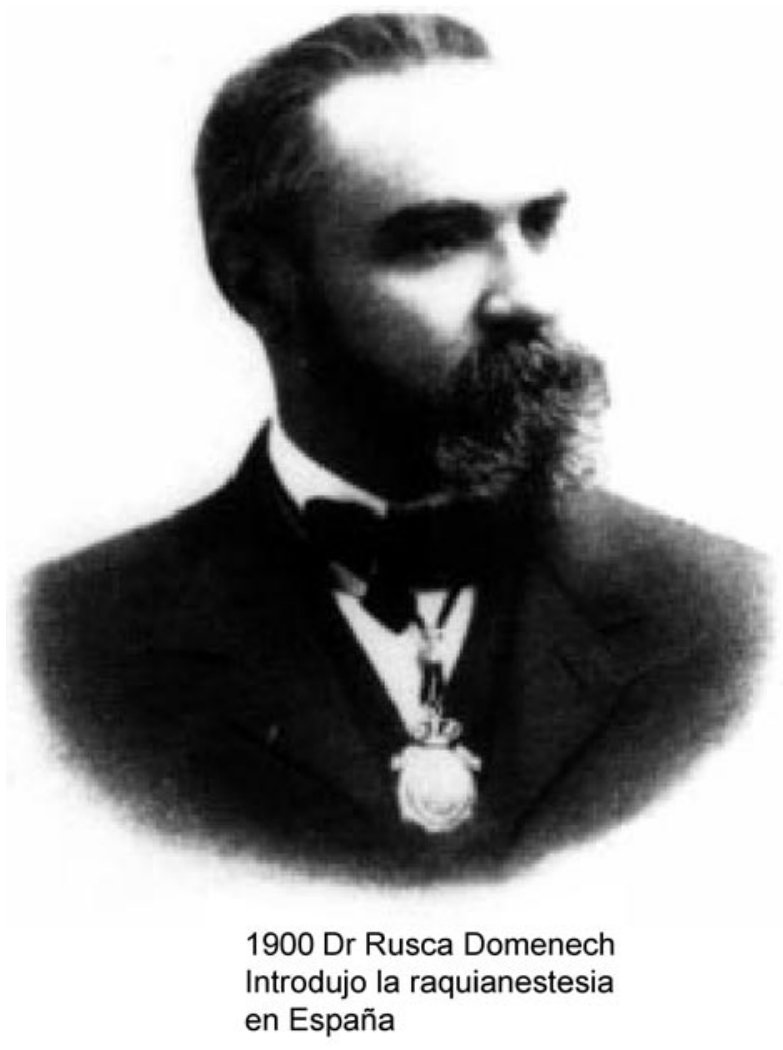

FIGURA 3. Francisco Rusca Doménech (1 868-1909).

anestesia intradural. La primera de ellas es la del Prof. Dr. J. M. Batrina (1877-1950), con una experiencia de 750 casos.

Otra es la dirigida por el Prof. Dr. V. Sagarra Lascuraín (1848-1924), catedrático de anatomía topográfica y técnica operatoria en la Facultad de Medicina de Valladolid, quien realiza una modificación

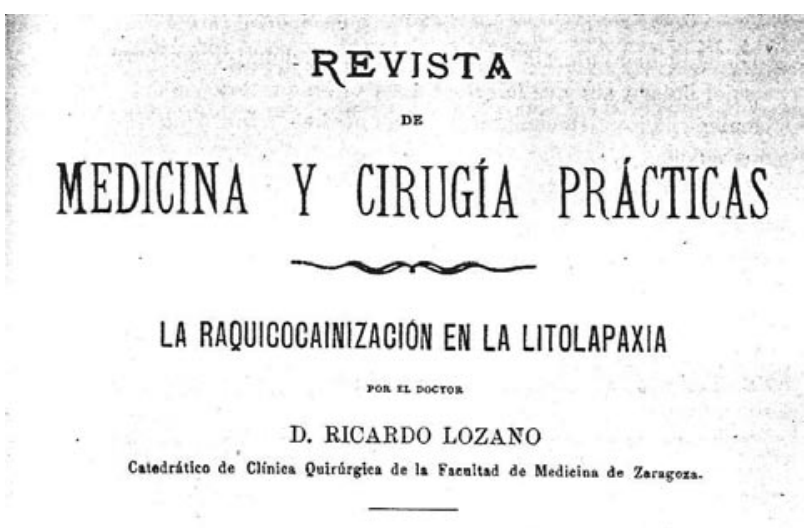

FIGURA 4. Artículo del Dr. Lozano de Zaragoza del uso de la raquicocainización. de la técnica de Jonnesco administrando una mezcla compuesta de un gramo de agua esterilizada, siete centígramos de estovaína y un miligramo de estricnina, caracterizada por una baja toxicidad y un buen poder anestésico.

Finalmente debemos hacer mención de otra importante escuela de la anestesia raquídea que es la dirigida por el Dr. Gómez Ulla (1877-1945) del Hospital Militar General de Carabanchel (Madrid). Llevo a cabo numerosas investigaciones con el fin de obtener mezclas estériles de anestésicos y sustancias estabilizadoras a las que denomino "ampollas raqui" que estaban constituidas por cloruro sódico hipertónico, estovaína, acido láctico y estricnina.

De esta manera a lo largo de la segunda década del siglo XX va aumentando el interés de los cirujanos españoles por el empleo de la anestesia raquídea pasando a ser el tema central de las comunicaciones a congresos médicos, de las discusiones en las academias de medicina y de numerosas tesis doctorales de la época (Figura 5).

\section{ANESTESIA EPIDURAL}

La anestesia epidural también denominada extradural o peridural, consiste en la inyección de la solución anestésica en el espacio comprendido entre la duramadre y el canal espinal. Se trata de un espacio continuo, de forma que en función del volumen de anestésico administrado y de su difusión así será el nivel de analgesia obtenido.

En lo que respecta a la aplicación de esta técnica debemos distinguir dos periodos, el primero de ellos de 1901-1920, en el que el único acceso al

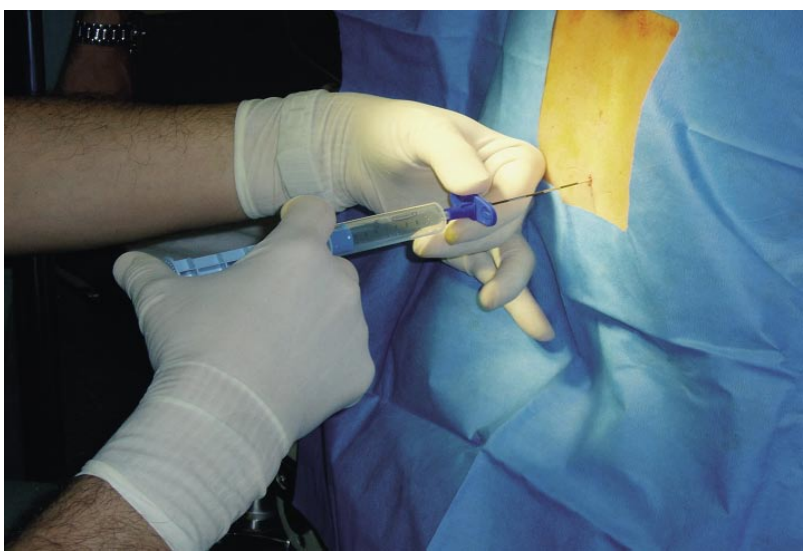

FIGURA 5. Método de punción en la anestesia raquídea. 
canal espinal se realizaba a través del hiato sacro, y un segundo periodo a partir de 1921 en el que se lleva a cabo la técnica mediante un abordaje lumbar o torácico.

\section{Anestesia a través del canal sacro}

El hiato sacro es el orificio inferior del conducto sacro situado a nivel de la III-IV vértebras sacras, cuyo interior es recorrido por el filum terminale. El empleo por primera vez de este acceso para la administración de anestesia se debe al urólogo francés Jean Athanase Sicard (1872-1929), en 1901. En el mismo año el cirujano francés Fernando Cathelin (1873-1945) realiza experimentos en animales con éxito pero no en sus pacientes, concluyendo que el grosor de las cubiertas de las raíces nerviosas sacras dificultaba la correcta difusión de los anestésicos.

En los siguientes años son muchos los cirujanos que emplean esta técnica con resultados muy dispares. En 1910 Oskar Gros (1877-1947) del Ins-

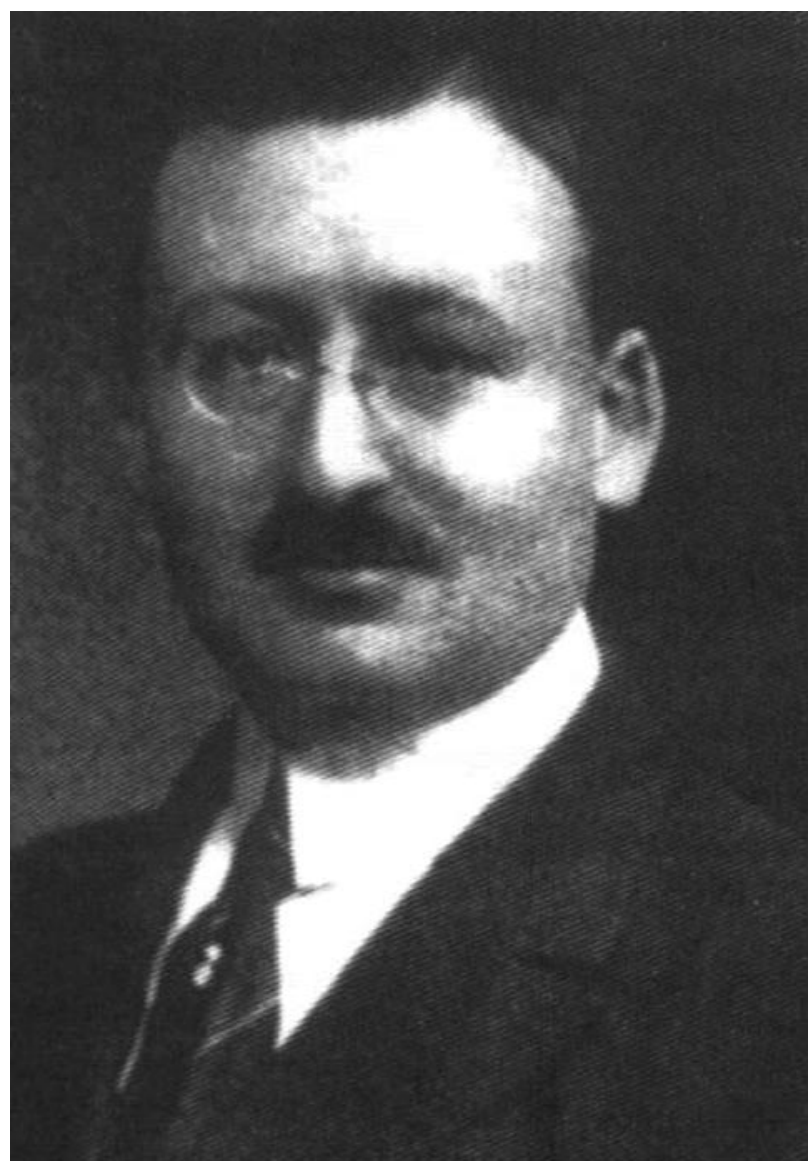

FIGURA 6. Fidel Pagés Miravé (1 886-1923). tituto de Farmacología de Leipzig (Alemania) describe como el poder de las soluciones anestésicas era mayor en medios alcalinos, empleando para ello bicarbonato sódico. Por su parte Arthur Läwen (18751958) describe en sus estudios el empleo epinefrina obteniendo una mezcla con menor absorción capilar pero mejor impregnación de los nervios por el anestésico.

A raíz de las publicaciones sobre la anestesia epidural se va empleando esta técnica para realizar de cirugía abdominal, cesáreas, cirugía renal, etc.

En 1917 el español S. Gil Vernet (18921987), profesor de anatomía y cirujano en Barcelona modifica la técnica de Läwen para conseguir un nivel más alto de anestesia. Realiza un estudio anatómico minucioso del canal sacro, describiendo que este canal mide $25 \mathrm{~mm}$ de ancho y el saco dural mide $10 \mathrm{~mm}$ y acaba a nivel de la segunda vértebra sacra, de forma que quedan dos espacios laterales de $7 \mathrm{~mm}$ cada uno aproximadamente ocupados por las raíces sacras, vasos sanguíneos y grasa. De esta forma se puede introducir una aguja hasta de $12 \mathrm{~cm}$ por este espacio sin perforar la duramadre inyectando la solución anestésica a un nivel más alto en el canal sacro. Gil Vernet publica numerosos artículos sobre su experiencia en 27 pacientes y en 1918 lee su tesis doctoral sobre este mismo tema.

En los años veinte y treinta la anestesia caudal se aplica de forma muy irregular. En torno al año 1940 comienzan a aparecer en la prensa médica estudios sobre la utilización, sobre todo en obstetricia, de catéteres ureterales que se introducían por el canal sacro dejándolos fijos con el fin de conseguir una analgesia continua, pudiendo añadir nuevas dosis de anestésico si el nivel de analgesia conseguido no era el suficiente. En España esta técnica fue introducida por los doctores J. M․ Bedoya y B. Pardo Ouro en 1944.

\section{Anestesia epidural lumbar o torácica}

En 1921 el cirujano español Fidel Pagés Miravé (1886-1923), desarrolla un nuevo método de anestesia epidural pinchando el canal espinal a nivel lumbar o torácico. Publicó su experiencia con 43 pacientes denominando a esta técnica anestesia metamérica, siendo el pionero mundial de la misma. Este procedimiento permite privar de sensibilidad un segmento del cuerpo, dejando con ella a las proporciones que están por encima y por debajo del segmento medular de donde proceden las raíces nerviosas bloqueadas. Su trabajo fue publicado en tres importan- 


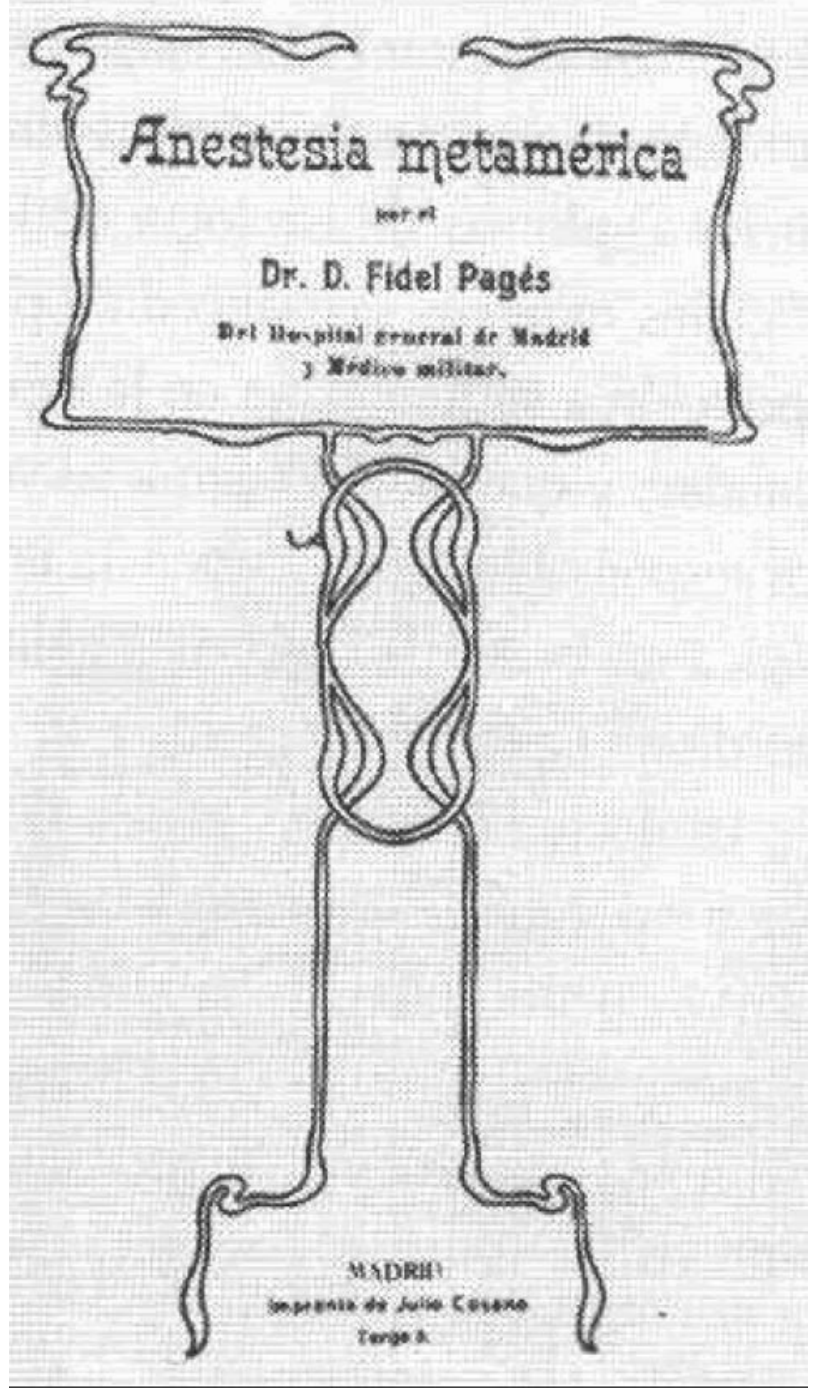

FIGURA 7. Libro sobre la Anestesia Metamérica de Fidel Pagés.

tes revistas españolas pero su muerte repentina en un accidente de coche en septiembre de 1923 hace que la técnica quede olvidada durante más de 10 años, privándole de dar difusión a su descubrimiento y de reclamar sus derechos a la paternidad del método. (Figuras 6, 7 y 8 ).

Diez años más tarde en 1931 el cirujano italiano Achile Mario Dogliotti (1897-1966), que suponemos desconocía los trabajos de Pagés, publica su método de la anestesia peridural lumbar. Doglioti tiene más suerte y sus trabajos se difunden por revistas médicas prestigiosas de numerosos países. En 1932 presenta una comunicación en el IX Congreso de la Sociedad Internacional de Cirugía en Madrid, pero nadie hace mención de lo trabajos de Pagés de 1921.

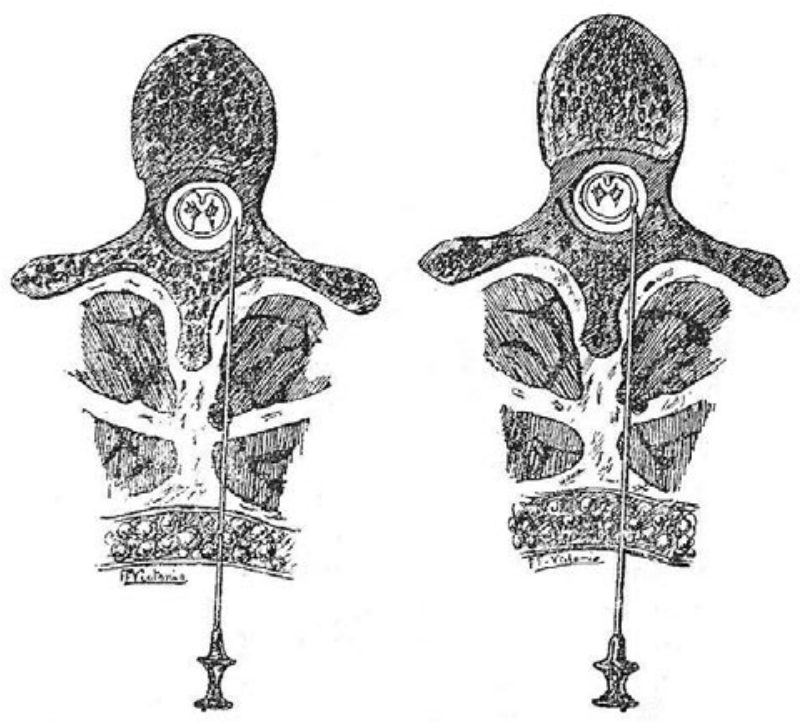

FIGURA 8. Esquema original del Dr. Pagés sobre la correcta colocación de la aguja en el canal espinal.

En 1932 el cirujano argentino Alberto Gutiérrez y el Dr. Tomás Rodríguez Mata fueron los primeros en recordar y en revindicar para Pagés la paternidad del método del la anestesia epidural. A partir de este momento la Academia de Cirugía de Madrid propuso denominar a la técnica anestesia epidural de Pagés - Dogliotti (Figura 9).

La anestesia epidural alta con acceso lumbar o torácico se va difundiendo por todo el mundo a lo largo de los años treinta. En España fue utilizada por J. M. Martínez Sagarra (1906-1992) y J. M. Remetería Aberasturi (1894-1970), ya en el año 1931. J.M. Martínez Sagarra publicó su trabajo sobre la

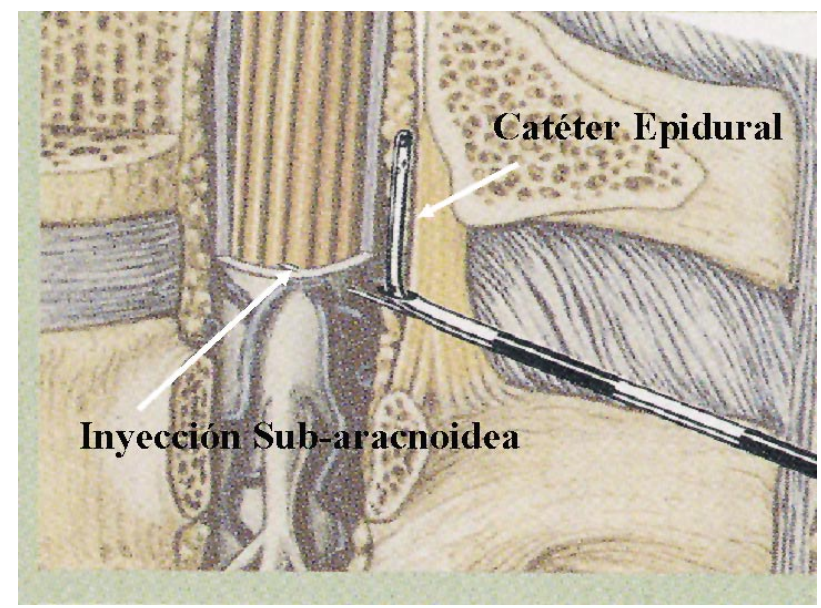

FIGURA 9. Anestesia epidural. Se introduce el catéter por fuera del ligamento amarillo. 
anestesia epidural en 1932 un mes antes de la realización del Congreso de la Sociedad Internacional de Cirugía en Madrid. Por su parte J.M. Remetería Aberasturi lleva a cabo las publicaciones en 1934 pero años antes ya había realizado investigaciones para modificar las dosis anestésicas recomendadas. Gracias a los trabajos de ambos la técnica de la anestesia epidural es difundida por España entre 1931 y 1936.

De esta forma la raquianestesia y la anestesia epidural se van extendiendo por todo el mundo y son utilizadas por multitud de cirujanos. A lo largo de los años se hacen nuevos avances con el diseño de catéteres, reservorios aguja... siendo empleadas estas técnicas no solo para intervenciones quirúrgicas sino en las clínicas de dolor para conseguir una analgesia continua.

\section{BIBLIOGRAFIA y LECTURAS RECOMENDADAS (*lectura de interés $y^{* *}$ lectura fundamental)}

*1. BARRAGÁN Y BONET, M.: "Anestesia quirúrgica producida por las inyecciones intrarraquídea de cocaína”, Rev. Med. Cirug. Pract., 49: 129, 1900.

2. BARTINA THOMÁS, J.M.: "Valeur de la Méthode d'Anesthesie par inyection intrarachidiennne". La Presse Medicale, 15, 1914.

**3. CORTÉS ROMAN, C.: "Anestesia epidural lumbar 1931-1936”. Segundo debut. Rev. Esp. Anestesiol. Reanim., 52: 159, 2005.
4. FRANCO GRANDE, A.; ÁlVAREZ ESCUDERO, J.; CORTÉS LIAÑO, J.: "Historia de la anestesia en España". Edit. Arán, Madrid, 2005.

**5. GIL VERNET, S.: "La anestesia extradural nueva técnica". Tip. S. Villalta, Barcelona, 1917.

6. GIL VERNET, S.: "Contribución al estudio de la anestesia local". Tesis doctoral mecanografiada, Madrid, 1918

*7. GONZÁLEZ RALERO, M.: "La anestesia raquídea en Urología". Rev. Esp. Cir. Urol., 13: 209, 1931.

8. LOZANO MONZÓN, R.: "La raquicocainización en la litolapaxia”. Rev. Med. Cirug. Pract., 54, 1902.

9. MARTÍN VIVALDI, J.: "Resultados obtenidos con la raquipercainización". Los Progresos de la Clínica, 44: 318, 1936.

10. OREJA ELÓSEGUI, B.: "Técnica de la prostatectomía y sus resultados". Rev. Esp. Cir. Urol., 10: 267, 1928.

11. PAGÉS MIRAVÉ, F.: "La anestesia metamérica". Rev. Esp. Cirug., 3: 3, 1921.

12. PASCUAL RÍOS, S.: "La anestesia raquídea en Urología”. La Medicina Ibera, 24: 328, 1929.

13. PÉREZ ALBACETE, M.: "100 Figuras de la Urología Española”. pág. 84-85, Tip., San Francisco, Murcia, 2005.

14. SAGARRA LASCURAIN, V.: "Contribución al estudio de la anestesia raquídea". El Siglo Médico, 62: 428, 1916.

15. SAGARRA, V.: "Consideraciones acerca del proceso de anestesia denominado Raqui-estrieno-estovainización de Jonnesco". La Clínica Castellana, 1: 252, 1911. 